\title{
Cumulative Social Risk and Cardiovascular Disease Among Adults in South Korea: A Cross-Sectional Analysis of a Nationally Representative Sample
}

\author{
Harold H. Lee, $\mathrm{PhD}^{1}$; Augustine W. Kang, $\mathrm{PhD}^{2}$; Hyunjoon Lee, $\mathrm{BS}^{3}$; Yoojin Cha, $\mathrm{ScM}^{2}$; Don Operario, $\mathrm{PhD}^{2}$
}

\begin{abstract}
Accessible Version: www.cdc.gov/pcd/issues/2020/19_0382.htm
Suggested citation for this article: Lee HH, Kang AW, Lee H, Cha Y, Operario D. Cumulative Social Risk and Cardiovascular Disease Among Adults in South Korea: A Cross-Sectional Analysis of a Nationally Representative Sample. Prev Chronic Dis 2020;17:190382. DOI: https://doi.org/10.5888/pcd17.190382.
\end{abstract}

\section{PEER REVIEWED}

\section{Summary}

What is already known on this topic?

The Framingham risk score is a robust algorithm to predict cardiovascular disease (CVD) risk based on demographic and clinical factors among European Americans.

What is added by this report?

The Framingham risk score was predictive of stroke, myocardial infarction, and angina among a nationally representative sample of adults in South Korea. In addition, cumulative social risk was predictive of CVD incidence, independently of Framingham risk score.

What are the implications for public health practice?

The effect of cumulative social risk on CVD may not be fully mediated by poor health behaviors (eg, smoking) and cardiometabolic profile (eg, blood pressure, diabetes, cholesterol). Further investigation of nonbiobehavioral mediators between social factors and CVD is warranted.

\section{Abstract}

\section{Introduction}

The Framingham risk score (FRS) is widely used to predict cardiovascular disease (CVD), but it neglects to account for social risk factors. Our study examined whether use of a cumulative social risk score in addition to the FRS improves prediction of CVD among South Korean adults.

\section{Methods}

We used nationally representative data on 19,147 adults aged 19 or older from the Korea National Health and Nutrition Examina- tion Survey 2013-2016. We computed a cumulative social risk score (range, $0-3$ ) based on 3 social risk factors: low household income, low level of education, and single-living status. CVD outcomes were stroke, myocardial infarction, and angina. Weighted logistic regression examined the associations between cumulative social risk, FRS, and CVD. McFadden pseudo- $R^{2}$ and area under receiver operating characteristic curve (AUC) assessed model performance. We conducted mediation analyses to quantify the association between cumulative social risk score and CVD outcomes that is not mediated by the FRS.

\section{Results}

A unit increase in social risk was associated with $89.4 \%$ higher risk of stroke diagnosis, controlling for the FRS $(P<.001)$. The FRS explained $8.0 \%$ of stroke diagnosis $\left(R^{2}\right)$ with fair discrimination $(\mathrm{AUC}=0.728$ ), and adding the cumulative social risk score enhanced $R^{2}$ and AUC by $2.4 \%$ and 0.039 . In the association between cumulative social risk and stroke, the proportion not mediated by the FRS was $65 \%(P<.001)$. We observed similar trends in myocardial infarction and angina, such that an increase in social risk was associated with increased relative risk of disease and improved disease diagnosis, and a large proportion of the association was not mediated by the FRS.

\section{Conclusion}

Controlling for the FRS, cumulative social risks predicted stroke, myocardial infarction, and angina among adults in South Korea. Future research is needed to examine non-FRS mediators between cumulative social risk and CVD.

\section{Introduction}

Cardiovascular disease (CVD) has been the leading cause of death in the past 20 years (1). In 2016, ischemic heart disease and stroke accounted for a combined 26\% (15.2 million) of global deaths (2). To curb the incidence of CVD, a comprehensive understanding of CVD etiology is needed. 
Epidemiological and biomedical research has made progress in understanding CVD etiology and the mechanisms through which determinants manifest as CVD. Among the better-known studies is the Framingham Heart Study, an ongoing longitudinal cohort study. Many known determinants of CVD, such as smoking, cholesterol, blood pressure, and physical activity, were first reported by the Framingham Heart Study (3-5). The Framingham risk score (FRS) was developed to predict a person's 10-year risk for CVD on the basis of demographic information, such as age and sex, and biobehavioral markers, such as cholesterol levels, smoking status, systolic blood pressure, and diabetes (6).

A limitation of the FRS is that it is based solely on individuallevel factors. Hence, it does not sufficiently account for the role of social factors in determining CVD risk. For example, empirical data suggest that the FRS underestimates CVD mortality and morbidity among adults with low socioeconomic status in the United States (7) and Scotland (8). A wealth of evidence corroborates the relationship between socioeconomic factors (household income, education, occupation, marital status, social support) on health, with some research even suggesting a causal influence $(9,10)$. Evidence on the social determinants of CVD has accumulated such that current scientific literature recommends including socioeconomic factors such as income, education, and social isolation in the FRS to enhance prediction of CVD $(11,12)$. Associations between socioeconomic status and CVD risk persist even after controlling for age, sex, smoking, hypertension, diabetes, physical activity, diet, cholesterol, and body weight (13). In 2 recent studies, CVD researchers examined composite measures of cumulative social risk $(14,15)$ by identifying indicators of social disadvantage that were consistently related to health outcomes, including household income, education, solitude (ie, whether one lives alone), and ethnicity $(14,16)$. In a nationally representative sample of US adults, cumulative social risk factors were associated with increased CVD mortality (15). An important gap in this research, however, is that we do not know whether cumulative social risk predicts CVD independently of the individual-level biobehavioral factors measured in the FRS.

Most evidence on CVD risk is based on participants sampled in the Western Hemisphere, despite studies indicating that the prevalence of chronic disease is increasing rapidly across the world, in nations such as South Korea (17). The economic growth in South Korea since the 1960s (18) has been accompanied by increases in inequalities in income and education. Concerns are emerging about health disparities in overall mortality and the prevalence of noncommunicable diseases (19-21).

The objective of our study was to examine the effect of cumulative social risk and the FRS on CVD incidence among adults in South Korea, with the following 3 aims. First, we investigated the association between the FRS and CVD incidence to assess the utility of the FRS among adults in South Korea. Second, we assessed the association between a cumulative social risk score (based on low household income, low level of education, and single-living status) and CVD incidence. Finally, we examined the association between the cumulative social risk score and CVD incidence, controlling for the FRS.

\section{Methods}

We analyzed nationally representative data from the 2013-2016 Korea National Health and Nutrition Examination Survey (KNHANES), conducted by the Korean Centers for Disease Control and Prevention (KCDC) and the Ministry of Health and Welfare. Data were collected from the publicly available KCDC website (http://knhanes.cdc.go.kr). A full description of KNHANES methodology is described elsewhere $(22,23)$. In brief, KNHANES is a nationally representative, cross-sectional sample of the noninstitutionalized South Korean population. The KNHANES team used a multistage clustered probability design based on the administrative district, place of residence, and residential means (ie, apartment, other than apartment) to ensure recruitment of a representative sample. KNHANES team members visited each sampled household, where they conducted physical examinations for the health survey and face-to-face interviews for the nutrition survey. In KNHANES 2013-2015, 29,321 persons were asked to participate, and 22,948 (78.3\%) agreed and responded to the survey. In KNHANES 2016, 10,806 were asked to participate, and 8,150 (75.4\%) agreed and responded to the survey. From 31,188 people who participated in the one-time survey, 12,041 were excluded because of missing variables (Figure). Our study sample consisted of 19,147 participants.

The opinions expressed by authors contributing to this journal do not necessarily reflect the opinions of the U.S. Department of Health and Human Services, the Public Health Service, the Centers for Disease Control and Prevention, or the authors' affiliated institutions. 


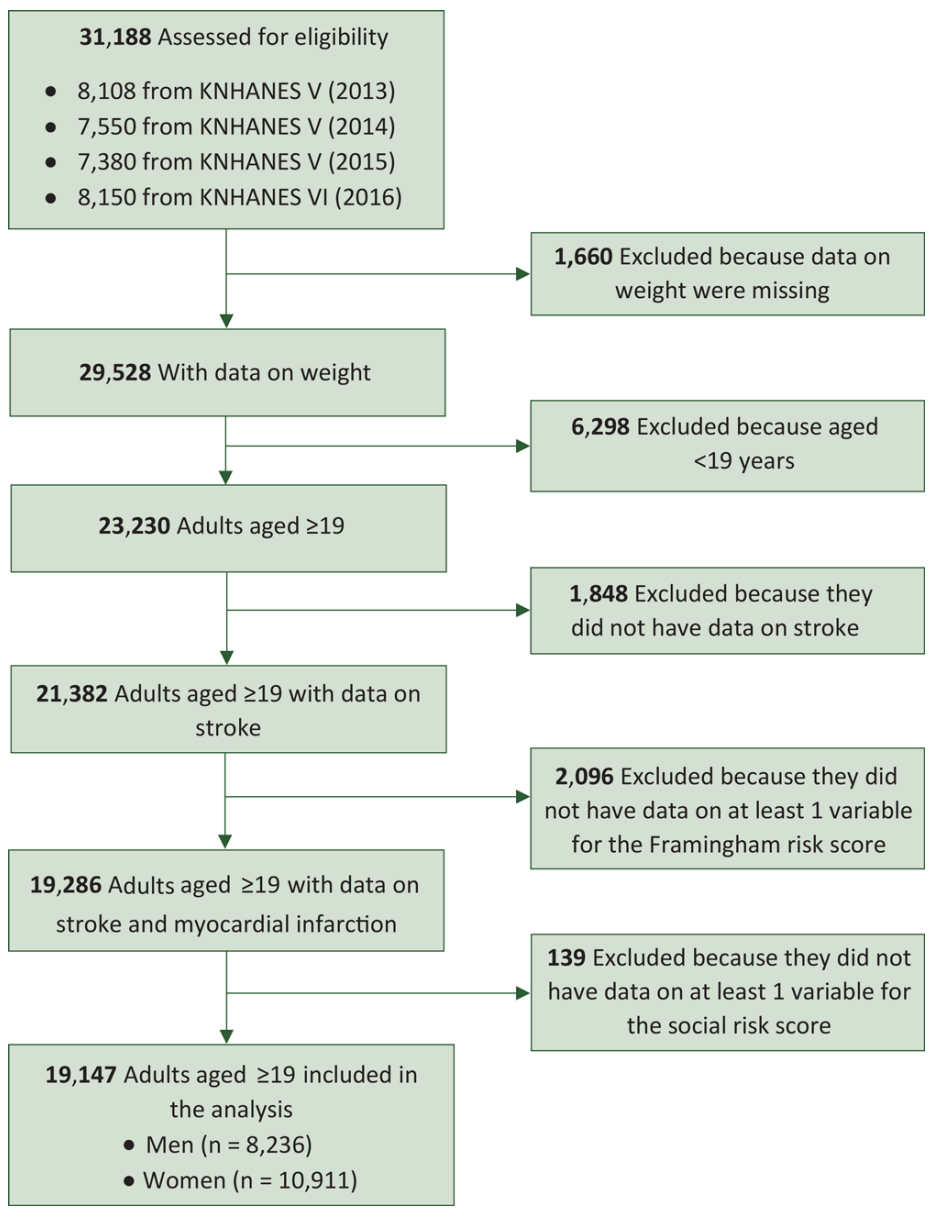

Figure. Potential participants included and excluded in the Korea National Health and Nutrition Examination Survey (KNHANES), 2013-2016.

Written informed consent was secured from all participants. The institutional review board of the KCDC approved this study, and the study protocol conformed to the ethical guidelines of the 1975 Declaration of Helsinki. The analysis was deemed exempt by the institutional review board of Brown University.

\section{Measures}

We computed the FRS using the algorithm described by D'Agostino et al (6). Components of the risk score are age, total cholesterol, high-density lipoprotein cholesterol, smoking status, diabetes status, systolic blood pressure, and whether or not the participant was currently being treated for hypertension. Consistent with previous studies $(14,15)$, we computed a cumulative social risk score (range, 0 to 3 ) by using 3 binary social risk factors: low household income (yes/no), low level of education (yes/no), and single-living status (yes/no). We did not include race or ethnicity in the cumulative social risk score because this information is not obtained by KNHANES, probably because South Korea is racially and ethnically homogeneous. KNHANES assessed household income by dividing monthly household income by the square root of the number of household members (adjusting for sex and each 5-year age stratum); participants were categorized into 4 quartiles of household income (upper, moderate, moderate-low, and low). We classified household income lower than the 50th percentile (ie, moderate-low and low) as low household income. KNHANES assessed education by using the question, "What is the highest qualification you obtained from school?" Response categories were college graduate, high school graduate, middle school graduate, and elementary school graduate. We classified middle-school graduate and elementary-school graduate as low level of education. KNHANES also assessed the number of people cohabitating per household by the question, "How many people are cohabiting with you?" Response categories ranged from 0 to 9 persons. Respondents who reported zero people were classified as single living. We examined 3 CVD outcomes: stroke, myocardial infarction, and angina. The occurrence of a lifetime CVD event was determined in the health interview survey in KNHANES. We classified participants who answered yes to the question, "Have you ever been diagnosed with (stroke, myocardial infarction, or angina) by a physician?" as persons with a previous CVD event.

\section{Analytic strategy}

We conducted all analyses in R version 3.3.1 (24), and we applied weights by using the svy command. First, we conducted descriptive analyses to examine distributions for all key variables. Second, we used weighted logistic regressions to examine the effect of the cumulative social risk score, controlling for the FRS. McFadden pseudo- $R^{2}$ assessed the variability of CVD diagnoses explained by predictors in each model. Goodness-of-fit ( $C$ statistic) assessed the area under the receiver operating characteristic curve (AUC). The AUC can range from 0.50 to 1.00 , with higher values indicating better predictive models. Although interpretation of AUC depends on context, a general guideline is that values above 0.80 indicate good models, between 0.70 and 0.80 fair models, and between 0.60 and 0.70 poor models (25). To adjust for multiple testing of 3 dependent variables (ie, stoke, myocardial infarction, and angina), we used a Bonferroni correction $\alpha$ of $0.016(0.05 / 3)$. Finally, we used mediation modeling to quantify the proportion not mediated by the FRS in the association between cumulative social risk and CVD outcomes. We assessed the mediator (ie, FRS) through simple linear regression (ie, FRS regressed on cumulative social risk) and logistic regression models (ie, CVD regressed on cumulative social risk with FRS as covariate). We then combined these results to estimate direct and indirect effects, using product-ofcoefficient methods (26). 


\section{Results}

The mean age of our KNHANES sample was 45.6 (standard deviation, 15.9) (Table 1). About three-quarters (76.9\%) of the sample were married, and $8.0 \%$ lived alone. The sample was well educated, with $76.0 \%$ of the sample having graduated from high school; $24.0 \%$ had a low level of education. More than one-third $(38.6 \%)$ had low household income. About half $(49.2 \%)$ had at least 1 social risk factor. Of the study sample, $1.6 \%$ reported having a stroke, $0.7 \%$ a myocardial infarction, and $1.4 \%$ angina.

A 1-unit increase in the cumulative social risk score was associated with $89.4 \%$ higher risk of having a stroke, controlling for the FRS $(P<.001)$ (Table 2$)$. The FRS alone explained $8.0 \%$ of the variance of a stroke diagnosis $\left(R^{2}\right)$, with fair discrimination (AUC $=0.728$ ). For this model, adding the cumulative social risk score improved $R^{2}$ by $2.4 \%$, and the AUC was increased by 0.039 . The shared contribution of the FRS and cumulative social risk score was $5.2 \%$, with a unique contribution of $2.8 \%$ from the FRS and a unique contribution of $2.4 \%$ from the cumulative social risk score, altogether predicting $10.4 \%$ variation of stroke diagnosis. In the association between cumulative social risk score and stroke, the proportion mediated by the FRS was $35 \%$ (95\% CI, 26\%-45\%, $P$ $<.001)$.

A 1-unit increase in the cumulative social risk score was associated with $62.7 \%$ higher risk of having a myocardial infarction, controlling for the FRS $(P<.001)$. The FRS alone explained 5.2\% of the variance of a stroke diagnosis $\left(R^{2}\right)$, with poor discrimination $(\mathrm{AUC}=0.674)$. For this model, adding the cumulative social risk score improved $R^{2}$ by $1.1 \%$, and the AUC was increased by 0.043 , resulting in fair discrimination $(A U C=0.713)$. The shared contribution of the FRS and the cumulative social risk score was $3.1 \%$, with a unique contribution of $2.1 \%$ from the FRS and a unique contribution of $1.1 \%$ from the cumulative social risk score, altogether predicting $6.3 \%$ variation of stroke diagnosis. In the association between the cumulative social risk score and myocardial infarction, the proportion mediated by the FRS was $40 \%(95 \% \mathrm{CI}$, $24 \%-62 \%, P<.001)$.

A 1-unit increase in the cumulative social risk score was associated with $63.6 \%$ higher risk of having angina, controlling for the FRS $(P<.001)$. The FRS alone explained $6.3 \%$ of the variance of an angina diagnosis $\left(R^{2}\right)$, with fair discrimination $(\mathrm{AUC}=0.734)$. For this model, adding the cumulative social risk score improved $R^{2}$ by $3.7 \%$, and the AUC was increased by 0.023 . The shared contribution of the FRS and cumulative social risk score was $4.9 \%$, with a unique contribution of $3.7 \%$ from the FRS and a unique contribution of $1.4 \%$ from the cumulative social risk score, altogether predicting $10.0 \%$ variation of angina diagnosis. In the association between the cumulative social risk score and angina, the proportion mediated by the FRS was 45\% (95\% CI, $35 \%-58 \%, P<.001)$.

\section{Discussion}

Cumulative social risk predicted 3 CVD outcomes (stroke, myocardial infarction, and angina) after controlling for the FRS and taking into consideration multiple testing. Our study demonstrated improved model prediction, which suggests that cumulative social risk may bear clinical significance.

To our knowledge, ours is the first study to examine cumulative social risk in relation to CVD independently of the FRS and the first to examine this relationship in a nationally representative sample of South Korean adults. Although no previous studies examined the relation between cumulative social risk and CVD controlling for the FRS, findings from our study are consistent with findings from previous studies that examined the association between socioeconomic status - a factor that largely overlaps with cumulative social risk - and CVD, controlling for the FRS. One study investigated the association between socioeconomic status (measured as $<12$ years of education or low income) and coronary heart disease, controlling for the FRS (7). Adding the socioeconomic status variable to a model with the FRS improved calibration, with predicted risk estimates of $3.1 \%$ for those with higher socioeconomic status and 5.2\% for those lower socioeconomic status; inclusion of socioeconomic status in the model resulted in upgrading risk classification for $15.1 \%$ of participants with low socioeconomic status. Similarly, in a prospective study of 12,304 men and women in western Scotland, the FRS underestimated CVD among manual workers, compared with nonmanual workers, and among people from deprived areas, compared with people from affluent areas (8).

In our study, cumulative social risk predicted CVD among adults in South Korea. Future comparative research is needed to examine whether the effect of cumulative social risk on CVD is stronger in South Korea than in the Western Hemisphere (eg, United States) or whether the effect is similar across contexts. Investigating the presence and size of this effect across contexts has public health relevance because such studies can inform whether interventions or policies to reduce CVD need to be culturally tailored according to geographical or cultural contexts. Although empirical studies appear to favor the idea that the link between cumulative social risk and CVD is a generalizable trend (14-16), research is needed to ascertain whether the findings from our study are replicable in other settings and to identify the mechanisms by which cumulative social risk leads to CVD.

\footnotetext{
The opinions expressed by authors contributing to this journal do not necessarily reflect the opinions of the U.S. Department of Health and Human Services, the Public Health Service, the Centers for Disease Control and Prevention, or the authors' affiliated institutions.
} 
In the association between cumulative social risk and CVD outcomes, the proportion mediated by the FRS was $35 \%$ to $45 \%$. Although this association corroborates the importance of intervening at the components of the FRS (eg, smoking, total cholesterol, blood pressure), it also highlights that a substantial proportion (ie, $\sim 55 \%-65 \%$ ) is not mediated by the FRS. Put differently, cumulative social risk may hypothetically influence CVD through nonbiobehavioral factors assessed in the FRS, such as increased levels of psychological stress (27) or decreased levels of optimism (28). Most empirical epidemiological studies that examined cumulative social risk on somatic health (eg, CVD, cancer, mortality) highlighted the possibility that cumulative social risk plays a key role in life-course epidemiology (eg, parenting and infant development, early childhood, transition to adulthood) (29). Taken together, research that examines the nonbehavioral pathways and developmental processes connecting cumulative social risk and CVD outcomes is warranted.

Our study has several strengths. First, it used a nationally representative sample of adults in South Korea. It had 3 CVD measures and found effects for all 3 outcomes. However, our study also had several limitations. KNHANES has a cross-sectional design, and, therefore, we cannot infer causality. Reverse causality is theoretically possible, whereby incidence of CVD (ie, unhealthiness) may cause low income, low levels of education, or solitude, although little empirical data exist to support these causal pathways. Because survey data were collected through interviews in participants' households, responses might have been affected by selfreport bias. Also, although the FRS encompasses some health behaviors (eg, smoking) and their downstream factors (eg, hypertension, diabetes), in the mediation analyses, we did not control for health behaviors that could lie in the pathway between cumulative social risk score and CVD, such as physical activity and diet (30). Although KNHANES asked participants whether CVD had been diagnosed by a physician, these data were self-reported. In addition, most types of angina may be classified as a CVD outcome, but our data did not differentiate between CVD-related angina and non-CVD-related angina. Angina may not be related to CVD if a person has no documented CVD or no risk factors for CVD.

The FRS predicted stroke, myocardial infarction, and angina in a nationally representative sample of South Korean adults. Moreover, a cumulative social risk measure that incorporated income, education, and single living predicted CVD independently of the FRS, suggesting that the potential effect of cumulative social risk on CVD may not be fully mediated by biological and behavioral markers assessed by the FRS (eg, smoking, blood pressure, diabetes, cholesterol). Future investigation is warranted to understand the nonbiobehavioral mediators (ie, factors not included in the FRS) between social risk and CVD.

\section{Acknowledgments}

H.H.L. was supported by the National Heart, Lung, and Blood Institute, National Institutes of Health (1F31HL140817). A.W.K. was supported by American Heart Association (18PRE34060015). D.O. was supported by the National Institute of Alcohol Abuse and Alcoholism, National Institutes of Health (U24 AA022000). We have no relationships that could be construed as a conflict of interest. We thank Dr Steven Worthington for his consultation on statistical analyses. No copyrighted materials were used in the conduct of this research or the writing of this article.

\section{Author Information}

Corresponding Author: Harold H. Lee, PhD, Department of Social and Behavioral Sciences, Harvard T.H. Chan School of Public Health, 401 Park Dr, 428F, Boston, MA 02215. Telephone: 951818-4246. Email: hhlee@hsph.harvard.edu.

Author Affiliations: ${ }^{1}$ Deparment of Social and Behavioral Sciences, Harvard T.H. Chan School of Public Health, Boston, Massachusetts. ${ }^{2}$ Department of Behavioral and Social Sciences, Brown University School of Public Health, Providence, Rhode Island. ${ }^{3}$ Department of Computer Science, Brown University, Providence, Rhode Island.

\section{References}

1. Murray CJ, Lopez AD. Global mortality, disability, and the contribution of risk factors: Global Burden of Disease Study. Lancet 1997;349(9063):1436-42.

2. World Health Organization. The top 10 causes of death. 2014. https://www.who.int/news-room/fact-sheets/detail/the-top-10causes-of-death. Accessed April 2, 2020.

3. Kannel WB, Sorlie P. Some health benefits of physical activity. The Framingham Study. Arch Intern Med 1979; 139(8):857-61.

4. Kannel WB, McGee DL. Diabetes and cardiovascular disease. The Framingham study. JAMA 1979;241(19):2035-8.

5. Kannel WB, Wolf PA, McGee DL, Dawber TR, McNamara P, Castelli WP. Systolic blood pressure, arterial rigidity, and risk of stroke. The Framingham study. JAMA 1981 ; 245(12):1225-9.

6. D’Agostino RB Sr, Vasan RS, Pencina MJ, Wolf PA, Cobain M, Massaro JM, et al. General cardiovascular risk profile for use in primary care: the Framingham Heart Study. Circulation 2008;117(6):743-53.

The opinions expressed by authors contributing to this journal do not necessarily reflect the opinions of the U.S. Department of Health and Human Services, the Public Health Service, the Centers for Disease Control and Prevention, or the authors' affiliated institutions. 
7. Fiscella K, Tancredi D, Franks P. Adding socioeconomic status to Framingham scoring to reduce disparities in coronary risk assessment. Am Heart J 2009;157(6):988-94.

8. Brindle PM, McConnachie A, Upton MN, Hart CL, Davey Smith G, Watt GC. The accuracy of the Framingham risk-score in different socioeconomic groups: a prospective study. Br J Gen Pract 2005;55(520):838-45.

9. Evans GW, Kim P. Multiple risk exposure as a potential explanatory mechanism for the socioeconomic status-health gradient. Ann N Y Acad Sci 2010;1186(1):174-89.

10. Adler NE, Boyce T, Chesney MA, Cohen S, Folkman S, Kahn RL, et al. Socioeconomic status and health. The challenge of the gradient. Am Psychol 1994;49(1):15-24.

11. Seeman T, Merkin SS, Crimmins E, Koretz B, Charette S, Karlamangla A. Education, income and ethnic differences in cumulative biological risk profiles in a national sample of US adults: NHANES III (1988-1994). Soc Sci Med 2008; 66(1):72-87.

12. Tunstall-Pedoe H, Woodward M; SIGN group on risk estimation. By neglecting deprivation, cardiovascular risk scoring will exacerbate social gradients in disease. Heart 2006; 92(3):307-10.

13. Fiscella K, Franks P. Should years of schooling be used to guide treatment of coronary risk factors? Ann Fam Med 2004; 2(5):469-73.

14. Caleyachetty R, Echouffo-Tcheugui JB, Shimbo D, Zhu W, Muennig P. Cumulative social risk and risk of death from cardiovascular diseases and all-causes. Int J Cardiol 2014; 177(3):1106-7.

15. Caleyachetty R, Echouffo-Tcheugui JB, Muennig P, Zhu W, Muntner P, Shimbo D. Association between cumulative social risk and ideal cardiovascular health in US adults: NHANES 1999-2006. Int J Cardiol 2015;191:296-300.

16. Bauman LJ, Silver EJ, Stein RE. Cumulative social disadvantage and child health. Pediatrics 2006;117(4):1321-8.

17. Yoon K-H, Lee J-H, Kim J-W, Cho JH, Choi Y-H, Ko S-H, et al. Epidemic obesity and type 2 diabetes in Asia. Lancet 2006; 368(9548):1681-8.

18. Kuznets PW. An East Asian model of economic development: Japan, Taiwan, and South Korea. Econ Dev Cult Change 1988; 36(S3S3):S11-43.

19. Hwang J, Shon C. Relationship between socioeconomic status and type 2 diabetes: results from Korea National Health and Nutrition Examination Survey (KNHANES) 2010-2012. BMJ Open 2014;4(8):e005710.

20. Cha SH, Park HS, Cho HJ. Socioeconomic disparities in prevalence, treatment, and control of hypertension in middleaged Koreans. J Epidemiol 2012;22(5):425-32.
21. Khang Y-H, Kim HR. Explaining socioeconomic inequality in mortality among South Koreans: an examination of multiple pathways in a nationally representative longitudinal study. Int J Epidemiol 2005;34(3):630-7.

22. Kweon S, Kim Y, Jang MJ, Kim Y, Kim K, Choi S, et al. Data resource profile: the Korea National Health and Nutrition Examination Survey (KNHANES). Int J Epidemiol 2014; 43(1):69-77.

23. Kim Y. The Korea National Health and Nutrition Examination Survey (KNHANES): current status and challenges. Epidemiol Health 2014;36:e2014002.

24. R Development Core Team. The R project for statistical computing. 2016. https://www.r-project.org/. Accessed April $14,2020$.

25. Tape TG.Interpreting diagnostic tests: the area under an ROC curve. 2002. http://gim.unmc.edu/dxtests/ROC3.htm. Accessed September 15, 2019.

26. Rijnhart JJM, Twisk JWR, Eekhout I, Heymans MW. Comparison of logistic-regression based methods for simple mediation analysis with a dichotomous outcome variable. BMC Med Res Methodol 2019;19(1):19.

27. Steptoe A, Kivimäki M. Stress and cardiovascular disease: an update on current knowledge. Annu Rev Public Health 2013; 34(1):337-54.

28. Kim ES, Hagan KA, Grodstein F, DeMeo DL, De Vivo I, Kubzansky LD. Optimism and cause-specific mortality: a prospective cohort study. Am J Epidemiol 2017;185(1):21-9.

29. Burchinal M, Vernon-Feagans L, Cox M; Key Family Life Project Investigators. Cumulative social risk, parenting, and infant development in rural low-income communities. Parent Sci Pract 2008;8(1):41-69.

30. Lee HH, Pérez AE, Operario D. Age moderates the effect of socioeconomic status on physical activity level among South Korean adults: cross-sectional analysis of nationally representative sample. BMC Public Health 2019;19(1):1332.

\footnotetext{
The opinions expressed by authors contributing to this journal do not necessarily reflect the opinions of the U.S. Department of Health and Human Services, the Public Health Service, the Centers for Disease Control and Prevention, or the authors' affiliated institutions.
} 


\section{Tables}

Table 1. Population-Weighted Characteristics of the Study Sample, Adults Aged $\geq 19$ ( $n=19,147)$ From the Korea National Health and Nutrition Examination Survey (KNHANES), 2013-2016

\begin{tabular}{|c|c|}
\hline Characteristic & No. (Weighted \%) \\
\hline Age, mean (SE), y & $45.6(15.9)$ \\
\hline Female & $10,911(50.3)$ \\
\hline Married & $16,102(76.9)$ \\
\hline \multicolumn{2}{|l|}{ Social risk factors } \\
\hline Low household income $e^{a}$ & $8,223(38.6)$ \\
\hline Low level of education ${ }^{b}$ & $6,085(24.0)$ \\
\hline Single living ${ }^{c}$ & $1,852(8.0)$ \\
\hline \multicolumn{2}{|l|}{ Framingham risk factors (except age and sex) } \\
\hline Total cholesterol, mean (SE), mg/dL & $189.6(35.7)$ \\
\hline High-density lipoprotein cholesterol, mean (SE), mg/dL & $51.2(12.4)$ \\
\hline Has ever smoked & $7,076(40.3)$ \\
\hline Has diabetes & $2,240(9.5)$ \\
\hline Systolic blood pressure, mean (SE), $\mathrm{mm} \mathrm{Hg}$ & $116.7(15.8)$ \\
\hline Receives treatment for hypertension & $3,945(15.5)$ \\
\hline \multicolumn{2}{|l|}{ Occurrence of a lifetime CVD event ${ }^{d}$} \\
\hline Stroke & $420(1.6)$ \\
\hline Myocardial infarction & $172(0.7)$ \\
\hline Angina & $364(1.4)$ \\
\hline \multicolumn{2}{|l|}{ Cumulative social risk score $^{\mathrm{e}}$} \\
\hline 0 & $8,671(50.8)$ \\
\hline 1 & $5,790(31.0)$ \\
\hline 2 & 3,688 (15.1) \\
\hline 3 & 998 (3.1) \\
\hline
\end{tabular}

Abbreviation: CVD, cardiovascular disease.

${ }^{a}$ Participants were categorized into 4 quartiles of household income (upper, moderate, moderate-low, and low). Household income lower than the 50th percentile (ie, moderate-low and low) was categorized as low household income.

${ }^{\mathrm{b}}$ KNHANES asked, "What is the highest qualification you obtained from school?" Response categories were college graduate, high school graduate, middle school graduate, and elementary school graduate. Middle school graduate and elementary school graduate were classified as low level of education.

"KNHANES asked, "How many people are cohabiting with you?" Response categories ranged from 0 to 9 persons. Respondents who reported zero people were classified as single living.

' KNHANES asked, "Have you ever been diagnosed with [stroke, myocardial infarction, or angina] by a physician?" Respondents who answered yes were categorized as having a lifetime occurrence.

e Possible range, 0-3: 0, no social risk factors reported; 1, 1 social risk factor reported; 2, 2 social risk factors reported; 3, 3 social risk factors reported. 
Table 2. Weighted Logistic Regression of Cardiovascular Disease on Framingham Risk Score ${ }^{a}$ and Cumulative Social Risk ${ }^{b}$, Adults Aged $\geq 19$ ( $\left.n=19,147\right)$ From the Korea National Health and Nutrition Examination Survey, 2013-2016 ${ }^{\mathrm{C}}$

\begin{tabular}{|l|c|r|}
\hline Cardiovascular Disease & Unadjusted \\
\hline Stroke & $1.81(1.70-1.95)[<.001]$ & Adjusted $^{\mathbf{d}}$ \\
\hline Framingham risk score & $2.53(2.25-2.84)[<.001]$ & $1.42(1.31-1.53)[<.001]$ \\
\hline Cumulative social risk score & \multicolumn{2}{|l|}{$1.89(1.64-2.17)[<.001]$} \\
\hline Myocardial infarction & $1.66(1.51-1.83)[<.001]$ & $1.37(1.22-1.55)[<.001]$ \\
\hline Framingham risk score & $2.11(1.79-2.49)[<.001]$ & $1.63(1.31-2.01)[.002]$ \\
\hline Cumulative social risk score & & \\
\hline Angina & $1.88(1.72-2.06)[<.001]$ & $1.56(1.41-1.73)[<.001]$ \\
\hline Framingham risk score & $2.35(2.08-2.66)[<.001]$ & $1.64(1.42-1.89)[<.001]$ \\
\hline Cumulative social risk score &
\end{tabular}

${ }^{a}$ Computed by using the algorithm described by D'Agostino et al (6). Components of the risk score are age, total cholesterol, high-density lipoprotein cholesterol, smoking status, diabetes status, systolic blood pressure, and whether or not the participant was treated for hypertension.

${ }^{\mathrm{b}}$ Consistent with previous studies $(14,15)$, we computed a cumulative social risk score (range, 0 to 3 ) by using 3 binary social risk factors: low household income (yes/no), low level of education (yes/no), and single-living status (yes/no).

${ }^{c}$ All values are relative risk (95\% confidence interval) $[P$ value].

${ }^{d}$ In the adjusted model, each cardiovascular disease was regressed on the Framingham risk score and cumulative social risk. 